\title{
PLIN2 inhibits insulin-induced glucose uptake in myoblasts through the activation of the NLRP3 inflammasome
}

\author{
KYUNG-AH CHO and PETER B. KANG \\ Division of Pediatric Neurology, Department of Pediatrics, \\ University of Florida College of Medicine, Gainesville, FL 32610, USA
}

Received January 8, 2015; Accepted June 29, 2015

DOI: $10.3892 /$ ijmm.2015.2276

\begin{abstract}
Impaired lipid metabolism and inflammatory pathways have individually been implicated in the development of insulin resistance in skeletal muscle; however, little evidence is available to date linking the two in this context. In this study, we explored a potential molecular mechanism underlying insulin resistance in myoblasts mediated by the crosstalk between lipid accumulation and inflammatory pathways. We examined the influence of perilipin 2 (PLIN2), one of the most highly expressed lipid droplet-associated proteins in skeletal muscle, on glucose uptake and on the nucleotide-binding domain, leucine-rich repeat containing protein 3 (NLRP3) inflammasome in vitro. PLIN2 overexpression in $\mathrm{C} 2 \mathrm{C} 12$ cells led to an increased expression of NLRP3, caspase-1 and interleukin (IL)-1 $\beta$, along with an impaired insulin-induced glucose uptake. This defect was remedied by the RNAi-mediated knockdown of NLRP3 expression. We also found that insulin receptor substrate-1 (IRS-1), a component of insulin signaling, was negatively regulated by NLRP3 and IL-1 $\beta$, and that IL-1 $\beta$ inhibited insulin-induced glucose uptake in myoblasts. These results suggest that PLIN2 inhibits insulin-induced glucose uptake by activating NLRP3, caspase- 1 and IL-1 $\beta$, leading to a decreased IRS-1 expression. This study provides in vitro evidence supporting an association between lipid metabolism and inflammatory pathways in the pathogenesis of insulin resistance in skeletal muscle, and suggests potential therapeutic targets that warrant further investigation.
\end{abstract}

\section{Introduction}

Skeletal muscle accounts for the majority of insulin-stimulated glucose uptake and is thus a key organ for the development of insulin resistance. Disordered lipid metabolism is considered a major contributor to skeletal muscle insulin resistance (1).

Correspondence to: Dr Peter B. Kang, Division of Pediatric Neurology, Department of Pediatrics, University of Florida College of Medicine, PO Box 100296, Gainesville, FL 32610, USA

E-mail: pbkang@ufl.edu

Key words: PLIN2, insulin resistance, skeletal muscle, NLRP3 inflammasome
Impaired insulin signaling, as observed in subjects with obesity and type 2 diabetes, is strongly associated with an excess accumulation of triacylglycerols (TAGs) within muscle fibers in skeletal muscle (2-5). TAGs are mainly stored in neutral lipid droplets (LDs), coated by lipid droplet-associated proteins which are referred to as perilipins $(6,7)$. One of the 5 perilipins (PLINs), PLIN2, is a marker for LDs in human skeletal muscle, and the levels of intramuscular PLIN2 and triglycerides are closely correlated. Studies have reported increased TAG accumulation and lipid droplet formation when PLIN2 is overexpressed in vitro (8-10). Conversely, the knockdown of PLIN2 in macrophages has been shown to decrease the size and number of cellular lipids and LDs (8). A previous study using a rat model of diabetes demonstrated that increased levels of Plin2 in skeletal muscle correlated with insulin resistance when the rats were fed a high-fat diet (11). Plin2 antisense oligonucleotides have been shown to protect mice from insulin resistance when fed high-fat diets (12). These studies indicate a connection between PLIN2 dysregulation and the development of insulin resistance; however, the molecular mechanisms underlying this response are not yet fully understood.

Inflammation also contributes to the development of insulin resistance (13-15). The nucleotide-binding domain, leucine-rich repeat containing protein 3 (NLRP3) inflammasome is a group of protein complexes that sense a diverse set of host-derived stimuli, such as damage-associated molecular patterns (DAMPs) and control the production of important pro-inflammatory cytokines, such as interleukin (IL)-1 $\beta$ (16). The NRLP3 inflammasome enhances insulin resistance by triggering inflammation in adipose tissue in subjects with obesity $(16,17)$.

In this study, we found that PLIN2 overexpression led to the activation of the NLRP3 inflammasome in $\mathrm{C} 2 \mathrm{C} 12$ cells, providing evidence that the PLIN2 inhibition of insulin-induced glucose uptake is linked to both defects in lipid metabolism and to inflammation.

\section{Materials and methods}

Cell culture. C2C12 mouse myoblasts (American Type Culture Collection, Manassas, VA, USA) were cultured in Dulbecco's modified Eagle's medium (DMEM; Sigma Aldrich, St. Louis, MO, USA) supplemented with $20 \%$ fetal bovine serum (FBS; Atlanta Biologicals, Flowery Branch, GA, USA), penicillin $(100 \mathrm{U} / \mathrm{ml})$ and streptomycin $(100 \mu \mathrm{g} / \mathrm{ml})$ (both from 
Sigma-Aldrich). All cells were maintained in a $5 \% \mathrm{CO}_{2}$ incubator at $37^{\circ} \mathrm{C}$. After the cryopreserved cells were thawed, they were subcultured for at least 2 passages, and experiments were performed at $24 \mathrm{~h}$ post-seeding.

Preparation of PLIN2 expression constructs. Human PLIN2 cDNA, including the open reading frame and 3 ' untranslated region (UTR), was amplified using PfuTurbo DNA polymerase (Agilent Technologies, Santa Clara, CA, USA) with primers

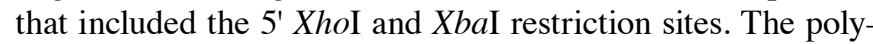
merase chain reaction (PCR) primers were as follows: forward, 5 '-ctcgagaagaaaaatggcatccgttgcagttgatcca-3' and reverse, 5 '-tctagaaactggtctatcctgcagtgaattttattgaattc-3'. The thermal cycling conditions were as follows: an initial denaturation step at $95^{\circ} \mathrm{C}$ for $2 \mathrm{~min}$, followed by 35 cycles of amplification $\left(95^{\circ} \mathrm{C}\right.$ for $30 \mathrm{sec}, 60^{\circ} \mathrm{C}$ for $30 \mathrm{sec}$, and $72^{\circ} \mathrm{C}$ for $2 \mathrm{~min}$ ), and a final extension at $72^{\circ} \mathrm{C}$ for $10 \mathrm{~min}$. Afterwards, an A nucleotide base overhang was generated by the addition of Ex Taq DNA polymerase (Clontech, Mountain View, CA, USA). The PCR product was inserted into the pGEM-T Easy Vector (Promega, Madison,WI, USA). For the construction of the mammalian expression vectors, TA clones were subcloned into the $X h o \mathrm{I}$ and $\mathrm{XbaI}$ sites in the $\mathrm{pCS}^{+}$vector, which was kindly provided by Dr Louis Kunkel (Division of Genetics and Genomics, Boston Children's Hospital and Harvard Medical School, Boston, MA, USA).

Cell transfection. To induce the overexpression of PLIN2 in the $\mathrm{C} 2 \mathrm{C} 12$ cells, the cells (at $70 \%$ confluence) were transfected with the $\mathrm{pCS} 2^{+}$vector expressing full-length human PLIN2 using Lipofectamine 2000 reagent (Life Technologies, Carlsbad, CA, USA) according to the manufacturer's instructions. An empty pCS2 ${ }^{+}$vector was transfected into the $\mathrm{C} 2 \mathrm{C} 12$ cells as a control. To reduce endogenous NLRP3 expression, the $\mathrm{C} 2 \mathrm{C} 12$ cells were transfected with 80 pmol of siRNA oligonucleotides (Santa Cruz Biotechnology, Inc., Dallas, TX, USA) using Lipofectamine 2000 reagent. Non-targeted siRNA oligonucleotides (Santa Cruz Biotechnology, Inc.) were used as controls. The $\mathrm{C} 2 \mathrm{C} 12$ cells were also co-transfected with the $\mathrm{pCS}^{+}$vector expressing PLIN2 and siRNA oligonucleotides targeting NLRP3. At $48 \mathrm{~h}$ post-transfection, the cells were harvested to extract the protein, and $25 \mu \mathrm{g}$ of protein were used for the detection of PLIN2 or NLRP3 by western blot analysis.

Western blot analysis. The cells were lysed in lysis buffer (1\% Triton X-100, $150 \mathrm{mM} \mathrm{NaCl}, 20 \mathrm{mM}$ Tris, $\mathrm{pH}$ 7.5) with protease inhibitor cocktail and phenylmethylsulfonyl fluoride (PMSF) (both from Santa Cruz Biotechnology, Inc.), and $25 \mu \mathrm{g}$ of protein were separated by electrophoresis. The resolved protein was transferred onto polyvinylidene fluoride (PVDF) membranes (Millipore, Bedford, MA, USA). The membranes were blocked with 5\% skim milk in Tris-buffered saline (TBS) containing 0.1\% Tween-20 (Thermo Fisher Scientific, Waltham, MA, USA) for $1 \mathrm{~h}$ to reduce non-specific binding and then incubated with antibodies to NLRP3 (sc-66846), caspase-1 (sc-56036), IL-1 $\beta$ (sc-7884), $\beta$-actin (sc-47778; all from Santa Cruz Biotechnologies, Inc.) and PLIN2 (ab52355; Abcam, Cambridge, UK) at $4^{\circ} \mathrm{C}$ overnight. After washing 3 times with TBS containing $0.1 \%$ Tween-20, the membranes were incubated with horseradish peroxidase (HRP)-conjugated secondary antibody (ab6728, ab97051; Abcam), and the signals were visualized using an enhanced chemiluminescence (ECL) kit (Thermo Fisher Scientific) on a ChemiDoc XRS ${ }^{+}$imaging system (Bio-Rad, Hercules, CA, USA).

Measurement of intracellular triglyceride levels. At $48 \mathrm{~h}$ posttransfection with either PLIN2-expressing vector or the empty vector, the $\mathrm{C} 2 \mathrm{C} 12$ cells were harvested in radioimmunoprecipitation (RIPA) lysis buffer (Santa Cruz Biotechnologies, Inc.). The intracellular triglyceride levels were measured in the cell lysates using a colorimetric triglyceride quantification kit (ab65336; Abcam) according to the manufacturer's instructions at a $570 \mathrm{~nm}$ wavelength on a microplate reader (BioTek Instruments, Inc., Winooski, VT, USA). C2C12 cells incubated with $400 \mu \mathrm{M}$ palmitic acid (Sigma-Aldrich) for $24 \mathrm{~h}$ were also subjected to this triglyceride assay as a positive control.

Glucose uptake assay. At $48 \mathrm{~h}$ post-transfection with either PLIN2-expressing vector or the empty vector, $\mathrm{C} 2 \mathrm{C} 12$ cells were washed twice with Krebs-Ringer-Phosphate-HEPES

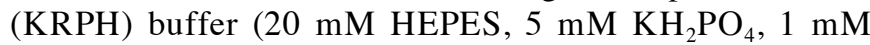
$\mathrm{MgSO}_{4}, 1 \mathrm{mM} \mathrm{CaCl}_{2}, 136 \mathrm{mM} \mathrm{NaCl}$ and $4.7 \mathrm{mM} \mathrm{KCl}, \mathrm{pH}$ 7.4) and starved of glucose by incubation with KRPH buffer containing $0.2 \%$ bovine serum albumin (BSA) at $37^{\circ} \mathrm{C}$ for $40 \mathrm{~min}$. The cells were then stimulated with $100 \mathrm{nM}$ insulin (Sigma-Aldrich) for $30 \mathrm{~min}$ in KRPH buffer supplemented with $0.2 \%$ BSA. Glucose transport was determined by subsequent stimulation with 2-deoxy-D-glucose-6-phosphate (2DG6P) at a final concentration of $0.1 \mathrm{mM}$ for $20 \mathrm{~min}$, as previously described (18). The reaction was terminated by washing the cells 4 times with ice-cold phosphate-buffered saline (PBS). The cells were lysed in lysis buffer, and glucose uptake was assessed using the Glucose Uptake Colorimetric assay kit (MAK083; Sigma-Aldrich) in accordance with the manufacturer's instructions. The absorbance was measured at a $412 \mathrm{~nm}$ wavelength on a microplate reader (BioTek Instruments, Inc.). To confirm the effects of IL-1 $\beta$ treatment on glucose uptake, the $\mathrm{C} 2 \mathrm{C} 12$ cells were incubated with murine IL-1 $\beta$ (PeproTech, Rocky Hill, NJ, USA) at 10,25 and $50 \mathrm{ng} / \mathrm{ml}$ for $24 \mathrm{~h}$. The cells were then subjected to the glucose uptake assay described above.

Reverse transcription PCR (RT-PCR). Total RNA was extracted from the $\mathrm{C} 2 \mathrm{C} 12$ cells in each experimental group using TRIzol reagent (Life Technologies). At $48 \mathrm{~h}$ post-transfection with PLIN2-overexpressing vector and a combination of PLIN2expressing vector with either NLRP3 siRNA or non-targeted control siRNA, the $\mathrm{C} 2 \mathrm{C} 12$ cells were stimulated with $100 \mathrm{nM}$ insulin for $30 \mathrm{~min}$. This was followed by RNA extraction to measure the expression levels of insulin receptor substrate-1 (IRS-1). RNA was also extracted from the $\mathrm{C} 2 \mathrm{C} 12$ cells treated with murine IL-1 $\beta$ at concentrations of 10,25 and $50 \mathrm{ng} / \mathrm{ml}$. First-strand cDNA was constructed with an oligo(dT) primer using the Superscript III first-strand synthesis system (Life Technologies) for RT-PCR. PCR was performed using Ex Taq DNA polymerase as follows: an initial denaturation step at $95^{\circ} \mathrm{C}$ for $2 \mathrm{~min}$, followed by 35 cycles of amplification $\left(95^{\circ} \mathrm{C}\right.$ for $30 \mathrm{sec}, 56^{\circ} \mathrm{C}$ for $30 \mathrm{sec}, 72^{\circ} \mathrm{C}$ for $1 \mathrm{~min}$ ) using the DNA Engine System (Bio-Rad). IRS-I (209 bp) was amplified using the following primers: forward, 5'-CGATGGCTTCTCAGA CGTG-3' and reverse, 5'-CAGCCCGCTTGTTGATGTTG-3'. The internal control gene, GAPDH (395 bp), was amplified 
A
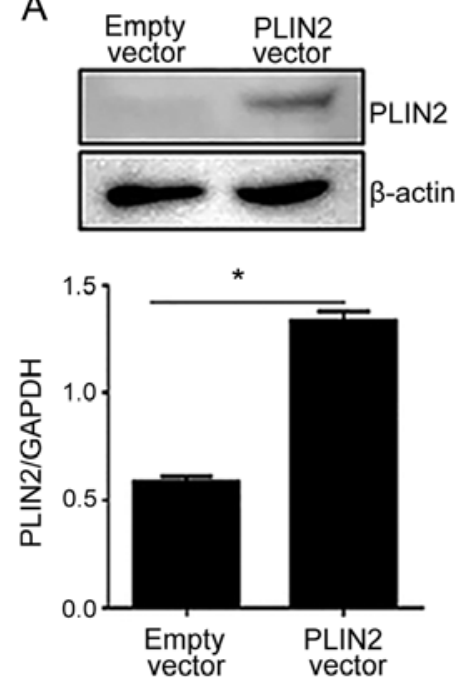

B
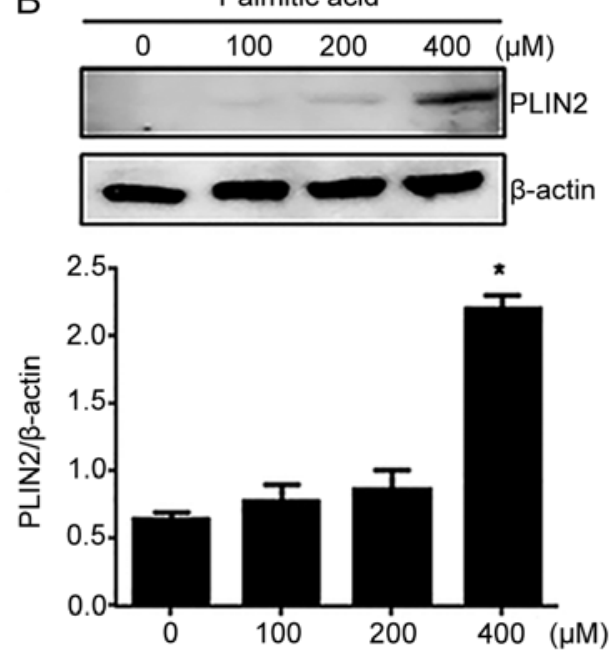

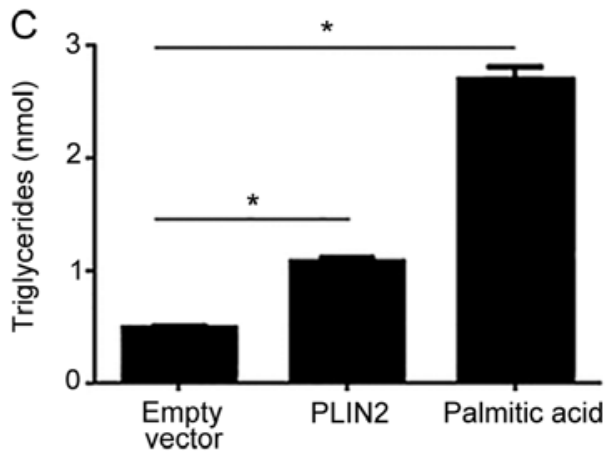

Figure 1. Perilipin 2 (PLIN2) induces triglyceride accumulation in C2C12 cells. (A) C2C12 cells transfected with empty vector or PLIN2-expressing vector were lysed in lysis buffer, and the cell lysates were assessed by western blot analysis. Histogram shows the protein expression of PLIN2, normalized to $\beta$-actin, as determined by densitometric analysis. (B) PLIN2 protein levels in C2C12 cells treated with $0,100,200$ and $400 \mu \mathrm{M}$ palmitic acid for 24 h. Cells treated with $400 \mu \mathrm{M}$ palmitic acid demonstrated a significant induction of PLIN2 in comparison with the cells treated with 0 , 100 or $200 \mu \mathrm{M}$ palmitic acid. The $\beta$-actin protein signal was used for normalization. (C) Triglyceride levels in C2C12 cells transfected with empty vector or PLIN2-expressing vector were examined. $\mathrm{C} 2 \mathrm{C} 12$ cells incubated with $400 \mu \mathrm{M}$ palmitic acid were used as positive controls. The data are expressed as the means $\pm \mathrm{SEM}$. ${ }^{*} \mathrm{P}<0.05$.

using the following primers: forward, 5'-GTC TTC TCC ACC ATG GAG AAG GCT-3' and reverse, 5'-CAT GCC AGT GAG CTT CCC GTT CA-3'.

Statistical analysis. Values are expressed as the means \pm standard error of the mean (SEM). Statistical significance was analyzed using the Student's t-test with GraphPad Prism 6 software (GraphPad Software Inc., San Diego, CA, USA). For all analyses, a value of $\mathrm{P}<0.05$ was considered to indicate a statistically significant difference.

\section{Results}

PLIN2 overexpression leads to the cellular accumulation of triglycerides. To determine the effects of PLIN2 on the accumulation of intracellular triglycerides, we overexpressed full-length human PLIN2 in the C2C12 cells using 2 methods: transient transfection and treatment with palmitic acid. Palmitic acid is a saturated fatty acid that activates peroxisome proliferator-activated receptor $\gamma$ (PPAR- $\gamma$ ), which in turn acts as a transcriptional activator of PLIN2 (19-21). Western blot analysis demonstrated a significant induction of PLIN2 expression following transfection with the PLIN2-overexpressing vector (Fig. 1A). Various concentrations (0, 100, 200 and $400 \mu \mathrm{M})$ of palmitic acid were used to mimic PLIN2 induction. Western blot analysis indicated that treatment of the C2C12 cells with $400 \mu \mathrm{M}$ of palmitic acid resulted in significantly higher PLIN2 levels compared to treatment with 0,100 or $200 \mu \mathrm{M}$ of palmitic acid (Fig. 1B). We then measured the intracellular triglyceride levels in the $\mathrm{C} 2 \mathrm{C} 12$ cells in which the overexpression of PLIN2 was induced using either method: transient transfection with the vector expressing PLIN2 or treatment with $400 \mu \mathrm{M}$ palmitic acid, the latter serving as a positive control. Both treatments yielded intracellular triglyceride levels that were significantly higher than those in the cells transfected with the empty vector; however, the cells treated with palmitic acid displayed a more dramatic elevation in intracellular triglyceride levels than the cells transfected with the PLIN2-overexpressing vector (Fig. 1C).

PLIN2 overexpression impairs insulin-stimulated glucose uptake. Since increased levels of intracellular triglycerides are associated with an impaired glucose uptake (22), we hypothesized that glucose uptake would be reduced in $\mathrm{C} 2 \mathrm{C} 12$ cells that overexpress PLIN2. Following transfection with the PLIN2-expressing vector, the $\mathrm{C} 2 \mathrm{C} 12$ cells were subjected to an insulin-stimulated glucose uptake assay. C2C12 cells transfected with the empty vector responded with a significant escalation of glucose uptake in response to insulin stimulation, whereas the C2C12 cells that overexpressed PLIN2 exhibited an impaired glucose uptake in response to insulin stimulation (Fig. 2A).

PLIN2 impairs insulin-stimulated glucose uptake activity through the activation of the NLRP3 inflammasome. We then investigated the potential involvement of the NLRP3 inflammasome in PLIN2-induced insulin resistance. This pathway has been implicated in recognizing certain non-microbial 'danger signals' that lead to caspase- 1 activation and the subsequent production of IL-1 $\beta$. As excessive fat is also known to activate the NLRP3 inflammasome (17), we hypothesized that this inflammasome would be activated by accumulating levels of triglycerides in $\mathrm{C} 2 \mathrm{C} 12$ cells that overexpress PLIN2. PLIN2 overexpression led to an increased protein expression of NLRP3, caspase-1 and IL-1 $\beta$, suggesting the activation of the NLRP3 inflammasome (Fig. 2B and C). To confirm that PLIN2 affects cellular glucose uptake through the activation of the NLRP3 inflammasome, we performed NLRP3 knockdown experiments by transfection with siRNA. siRNA targeting NLRP3 effectively reduced the endogenous expression of NLRP3 protein (Fig. 3A). Subsequently, the $\mathrm{C} 2 \mathrm{C} 12$ cells were co-transfected with 

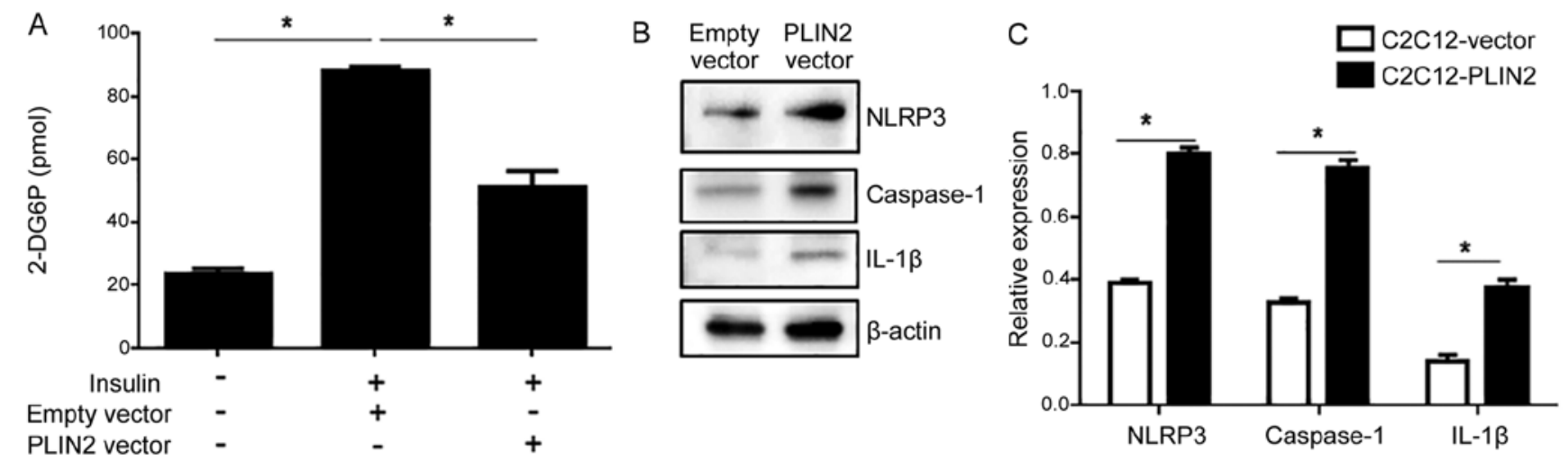

Figure 2. Perilipin 2 (PLIN2) overexpression impairs insulin-induced glucose uptake but activates the nucleotide-binding domain, leucine-rich repeat containing protein 3 (NLRP3) inflammasome. (A) C2C12 cells were transfected with either the empty vector or PLIN2-expressing vector and incubated with 100 nM insulin for $30 \mathrm{~min}$. After a 20-min pulse with 2-deoxy-D-glucose-6-phosphate (2-DG6P), glucose uptake was assessed. C2C12 cells that overexpressed PLIN2 showed a significant decrease in glucose uptake. (B) Expression of NLRP3, caspase-1 and interleukin-1 $\beta$ (IL-1 $\beta$ ) in C2C12 cells overexpressing PLIN2 was assessed by western blot analysis. (C) Densitometric analysis of (B). The data are expressed as the means \pm SEM. ${ }^{*} \mathrm{P}<0.05$.

PLIN2-expressing vector and NLRP3 siRNA, followed by a glucose uptake assay. NLRP3 knockdown resulted in decreased levels of NLRP3, caspase-1 and IL-1 $\beta$ in the cells overexpressing PLIN2 (Fig. 3B), and increased insulin-stimulated glucose uptake (Fig. 3C). The non-targeted control siRNA did not increase glucose uptake in the cells that overexpressed PLIN2.

Suppression of IRS-1 by IL-1 $\beta$ mediates the effects of the NLRP3 inflammasome on insulin-induced glucose uptake. IRS-1, one of the major substrates of the insulin receptor kinase, is essential for the activatation of PI3K in response to insulin, which leads to the phosphorylation of protein kinase B (also known as Akt) and subsequent glucose uptake (23). The downregulation of IRS-1 seems to be the major mechanism involved in the alteration of insulin signaling and glucose transport (24). Thus, we examined the effects of PLIN2 and NLRP3 manipulations on the mRNA expression levels of IRS- 1 in the C2C12 cells. PLIN2 overexpression (with transfection with control siRNA) led to a decrease in IRS-1 expression. However, PLIN2 overexpression accompanied by $N L R P 3$ knockdown via siRNA transfection was associated with an increased IRS- 1 expression in response to insulin stimulation (Fig. 3D). IL-1 $\beta$, the final step in the NLRP3 inflammasome, has been demonstrated to reduce insulin-induced glucose uptake in adipocytes by affecting IRS-1 expression $(18,25)$. To determine whether IL-1 $\beta$ treatment affects insulin-induced glucose uptake in myoblasts, we treated the $\mathrm{C} 2 \mathrm{C} 12$ cells with IL- $1 \beta$ at concentrations of 0,10 , 25 and $50 \mathrm{ng} / \mathrm{ml}$ for $24 \mathrm{~h}$ prior to measuring insulin-induced glucose uptake. IL-1 $\beta$ was found to inhibit glucose transport upon insulin stimulation in a dose-dependent manner (Fig. 4A). We also found that IL- $1 \beta$ decreased endogenous IRS- 1 expression in the C2C12 cells (Fig. 4B).

\section{Discussion}

Skeletal muscle is a major organ which is involved in insulin-stimulated glucose uptake and thus in the development of insulin resistance. Previous studies have demonstrated that excess lipid accumulation contributes to the pathogenesis of insulin resistance, thus suggesting potential therapeutic interventions $(2,26,27)$. Aside from the lipid overload hypothesis, inflammation has been considered to be a pivotal contributor to insulin resistance. Pro-inflammatory cytokines, such as tumor necrosis factor- $\alpha$ (TNF- $\alpha$ ), IL- 6 and monocyte chemoattractant protein-1 (MCP-1) have been identified as promoters of insulin resistance; however, these findings were mainly related to adipose tissue $(13,28)$.

In this study, we attempted to identify an association between lipid accumulation and the inflammatory pathway that leads to insulin resistance in $\mathrm{C} 2 \mathrm{C} 12$ cells. We found that the knockdown of NLRP3 attenuated PLIN2-induced insulin resistance in $\mathrm{C} 2 \mathrm{C} 12$ cells. PLIN2 is a lipid droplet-associated protein that is abundantly expressed in skeletal muscle. It is well known that the expression of PLIN2 correlates with the accumulation of intramuscular lipids (8-10). We observed in this study that $\mathrm{C} 2 \mathrm{C} 12$ cells which overexpressed PLIN2 contained significantly greater amounts of triglycerides. Furthermore, we also noted that PLIN2 overexpression led to the upregulation of IL-1 $\beta$ through NLRP3-caspase-1 upregulation.

The NLRP3 inflammasome consists of a group of protein complexes that are composed of the Nod-like receptor protein NLRP3 (also termed cryopyrin or NALP3), adapter proteins, a caspase recruitment domain (Cardinal) and caspase-1. The NLRP3 inflammasome has been implicated in the production of mature IL-1 $\beta$ and IL-18 in response to a variety of signals $(16,17)$. One of the signals, DAMPs, is host-derived and is released as a result of perturbations of tissue homeostasis caused by microbial or non-microbial insults, conveying a general sense of tissue under stress $(16,17)$. Although the normal activation of the NLRP3 inflammasome contributes to host defense, excessive activation results in inflammatory diseases mediated by the pro-inflammatory cytokine, IL-1 $\beta(16,17)$. It has been previously reported that the NLRP3 inflammasome instigates obesity-induced autoinflammation and insulin resistance (29). Considering that muscle is a critical organ involved in insulin sensitivity, we can speculate that the NLRP3 inflammasome links excess fat and insulin resistance in myoblasts. In the present study, we demonstrated that $\mathrm{C} 2 \mathrm{C} 12$ cells overexpressing PLIN2 exhibited increased levels of NLRP3, caspase-1 and IL-1 $\beta$. As PLIN2 impaired glucose uptake activity, this indicates a potential correlation between NLRP3 and glucose uptake in $\mathrm{C} 2 \mathrm{C} 12$ cells in response to insulin. The knockdown of NLRP3 
A

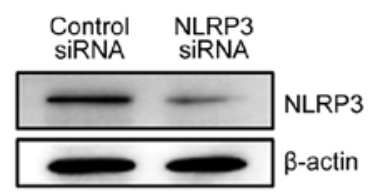

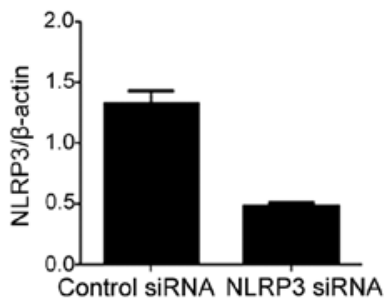

B

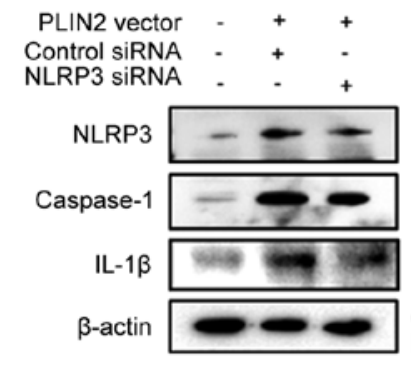

C
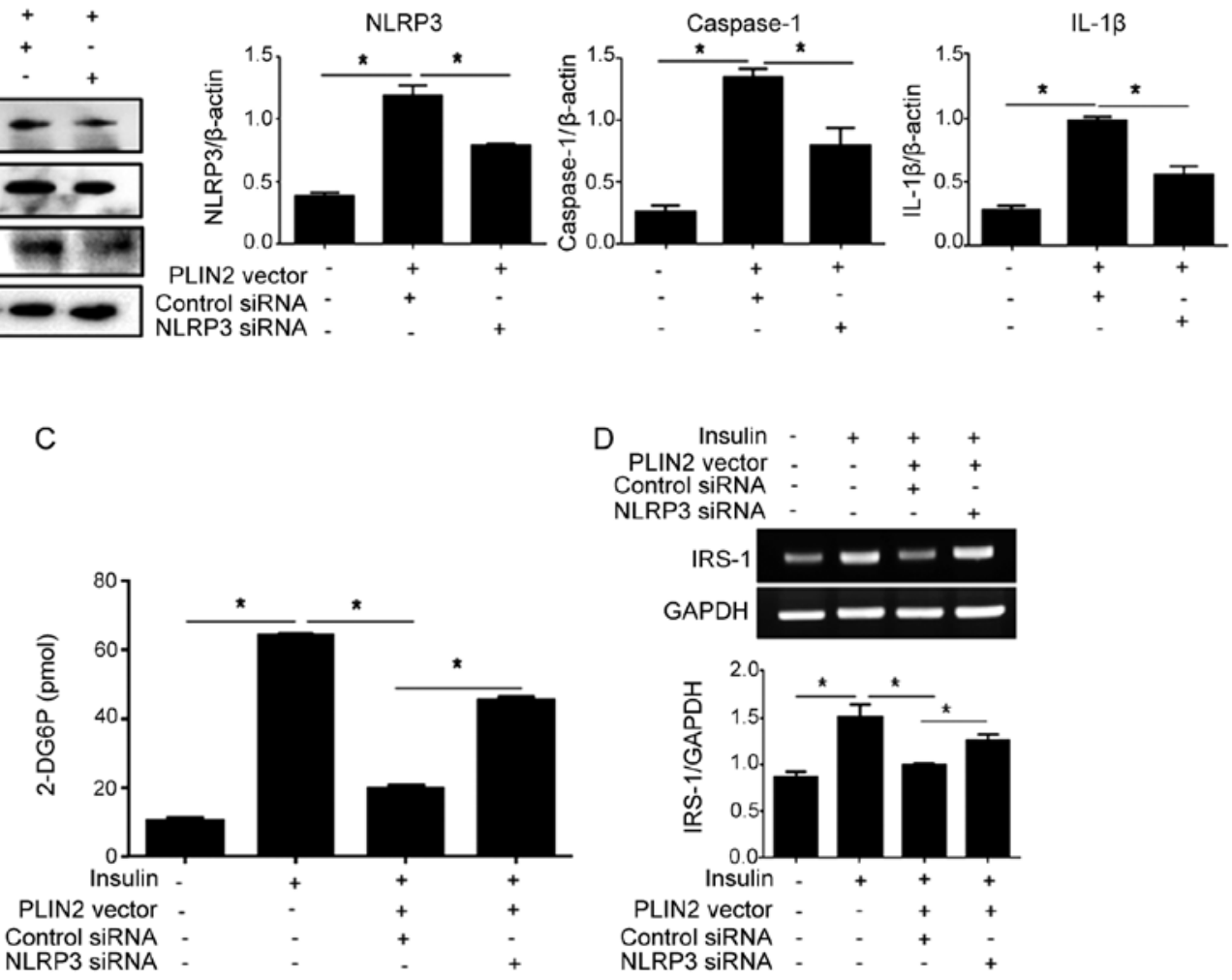

Figure 3. Nucleotide-binding domain, leucine-rich repeat containing protein 3 (NLRP3) silencing inhibits perilipin 2 (PLIN2)-induced insulin resistance. (A) Endogenous NLRP3 expression was reduced by targeted siRNA knockdown and quantified by western blot analysis. (B) Expression of NLRP3, caspase-1 and interleukin-1 $\beta$ (IL-1 $\beta$ ) after NLRP3 silencing in PLIN2 overexpressing C2C12 cells. Histograms represent densitometric analysis. (C) Glucose uptake was measured in $\mathrm{C} 2 \mathrm{C} 12$ cells from each experimental group. $\mathrm{C} 2 \mathrm{C} 12$ cells subjected to NLRP3 knockdown exhibited a recovery of glucose uptake along with PLIN2 overexpression. (D) mRNA expression of insulin receptor substrate-1 (IRS-1) in C2C12 cells from each experimental group was confirmed by endpoint PCR IRS-1 expression was decreased in PLIN2-overexpressing C2C12 cells exposed to insulin, whereas NLRP3 knockdown increased the levels of IRS-1. The data are expressed as the means \pm SEM.*P $<0.05$. 2-DG6P, 2-deoxy-D-glucose-6-phosphate.

A

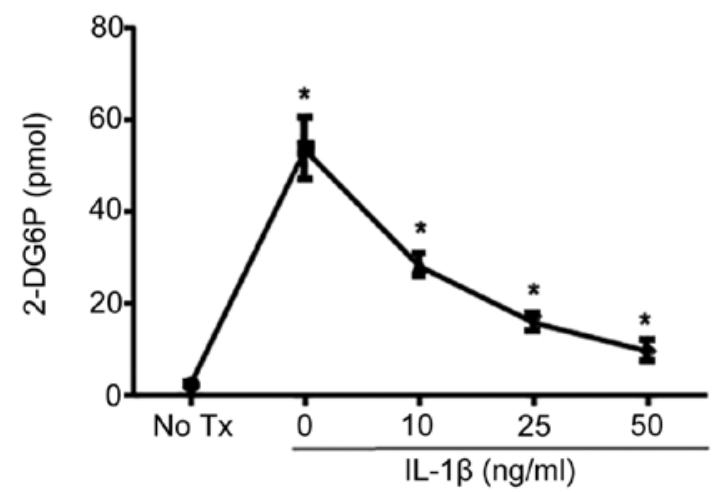

B
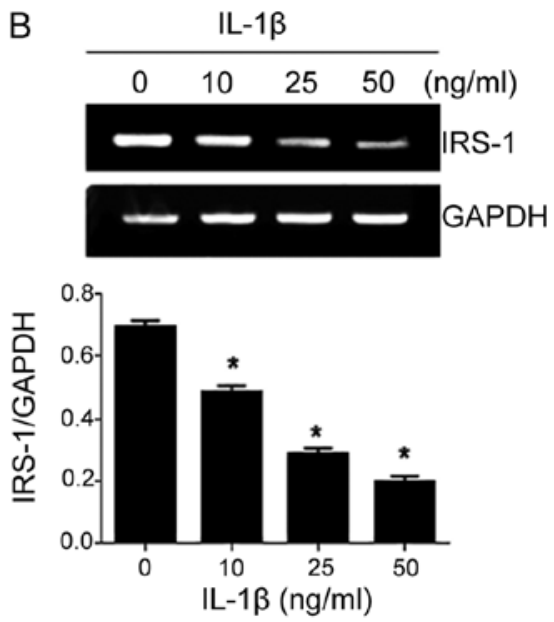

Figure 4. Interleukin-1 $\beta$ (IL-1 $\beta$ ) impairs the glucose uptake in response to insulin in $\mathrm{C} 2 \mathrm{C} 12$ cells and the expression of insulin receptor substrate-1 (IRS-1). (A) $\mathrm{C} 2 \mathrm{C} 12$ cells were treated with IL- $1 \beta$ at concentrations of 10,25 and $50 \mathrm{ng} / \mathrm{ml}$ for $24 \mathrm{~h}$, and insulin-induced glucose transport was then measured. (B) $\mathrm{mRNA}$ expression of IRS- 1 was confirmed in C2C12 cells incubated with IL- $1 \beta$ at concentrations of $10,25 \mathrm{and} 50 \mathrm{ng} / \mathrm{ml}$ for $24 \mathrm{~h}$. The histogram indicates densitometric analysis. The data are expressed as the means $\pm \mathrm{SEM}$. ${ }^{*} \mathrm{P}<0.05$. 2-DG6P, 2-deoxy-D-glucose-6-phosphate. No Tx, No treatment. 
by RNA interference significantly inhibited insulin resistance which is typically induced in PLIN2-overexpressing C2C12 cells, suggesting that the NLRP3 inflammasome mediates PLIN2-induced insulin resistance. Decreased levels of IRS-1 have been reported to alter insulin signaling in adipocytes and myocytes $(24,25)$. In this study, we observed that IRS-1 expression was decreased in $\mathrm{C} 2 \mathrm{C} 12$ cells that overexpressed PLIN2 in the context of insulin exposure, whereas NLRP3 knockdown increased the expression of IRS-1. IL-1 $\beta$, the final product of the NLRP3 inflammasome, has been reported to reduce insulin-induced glucose uptake in adipocytes, mainly by inhibiting IRS-1 expression (18). In the present study, we observed the inhibitory effect of IL- $1 \beta$ on the expression of IRS- 1 in $\mathrm{C} 2 \mathrm{C} 12$ cells, and on this basis we suggest that inflammatory cytokines directly target muscle cells, as well as adipocytes in the context of insulin signaling disturbances.

In conclusion, the present study provides a fresh view of the role of LDs and associated proteins in cellular metabolism by demonstrating the following novel insights. First, insulin-mediated cellular glucose uptake inversely correlated with PLIN2 expression in myoblasts. Second, components of the NLRP3 inflammasome were significantly upregulated in PLIN2-overexpressing myoblast cells, while the downregulation of NLRP3 inhibited PLIN2-induced insulin resistance. Taken together, these data suggest that PLIN2 influences cellular glucose uptake and transport by interacting with the NLRP3 inflammasome. Since insulin resistance can be characterized by inefficient glucose uptake in muscle and fat cells, our findings provide a molecular mechanism that links cellular lipid contents and the inflammatory response underlying the pathogenesis of insulin resistance. Future studies using animals fed a high-fat diet may help determine whether the suppression of the activation of the NLRP3 inflammasome would protect the animals against type 2 diabetes mellitus.

\section{Acknowledgements}

The authors thank the Department of Pediatrics at the University of Florida College of Medicine for supporting this study.

\section{References}

1. Rachek LI: Free fatty acids and skeletal muscle insulin resistance. Prog Mol Biol Transl Sci 121: 267-292, 2014.

2. Moors CC, van der Zijl NJ, Diamant M, Blaak EE and Goossens GH: Impaired insulin sensitivity is accompanied by disturbances in skeletal muscle fatty acid handling in subjects with impaired glucose metabolism. Int J Obes 36: 709-717, 2012.

3. Sinha R, Dufour S, Petersen KF, LeBon V, Enoksson S, Ma YZ, Savoye M, Rothman DL, Shulman GI and Caprio S: Assessment of skeletal muscle triglyceride content by (1)H nuclear magnetic resonance spectroscopy in lean and obese adolescents: relationships to insulin sensitivity, total body fat, and central adiposity. Diabetes 51: 1022-1027, 2002.

4. Kaneko S, Iida RH, Suga T, Fukui T, Morito M and Yamane A: Changes in triacylglycerol-accumulated fiber type, fiber type composition, and biogenesis in the mitochondria of the soleus muscle in obese rats. Anat Rec (Hoboken) 294: 1904-1912, 2011.

5. Gray RE, Tanner CJ,Pories WJ, MacDonald KG and Houmard JA Effect of weight loss on muscle lipid content in morbidly obese subjects. Am J Physiol Endocrinol Metab 284: E726-E732, 2003.

6. Ducharme NA and Bickel PE: Lipid droplets in lipogenesis and lipolysis. Endocrinology 149: 942-949, 2008.

7. Martin S and Parton RG: Lipid droplets: A unified view of a dynamic organelle. Nat Rev Mol Cell Biol 7: 373-378, 2006.
8. Larigauderie G, Cuaz-Pérolin C, Younes AB, Furman C, Lasselin C, Copin C, Jaye M, Fruchart JC and Rouis M: Adipophilin increases triglyceride storage in human macrophages by stimulation of biosynthesis and inhibition of beta-oxidation. FEBS J 273: 3498-3510, 2006.

9. Fukushima M,Enjoji M,Kohjima M, SugimotoR, Ohta S, Kotoh K, Kuniyoshi M, Kobayashi K, Imamura M, Inoguchi T, et al: Adipose differentiation related protein induces lipid accumulation and lipid droplet formation in hepatic stellate cells. In Vitro Cell Dev Biol Anim 41: 321-324, 2005.

10. Listenberger LL, Ostermeyer-Fay AG, Goldberg EB, Brown WJ and Brown DA: Adipocyte differentiation-related protein reduces the lipid droplet association of adipose triglyceride lipase and slows triacylglycerol turnover. J Lipid Res 48: 2751-2761, 2007.

11. Minnaard R, Schrauwen P, Schaart G, Jorgensen JA, Lenaers E, Mensink M and Hesselink MK: Adipocyte differentiation-related protein and OXPAT in rat and human skeletal muscle: involvement in lipid accumulation and type 2 diabetes mellitus. J Clin Endocrinol Metab 94: 4077-4085, 2009.

12. Varela GM, Antwi DA, Dhir R, Yin X, Singhal NS, Graham MJ, Crooke RM and Ahima RS: Inhibition of ADRP prevents diet-induced insulin resistance. Am J Physiol Gastrointest Liver Physiol 295: G621-G628, 2008.

13. Maury E and Brichard SM: Adipokine dysregulation, adipose tissue inflammation and metabolic syndrome. Mol Cell Endocrinol 314: 1-16, 2010.

14. Sell H and Eckel J: Monocyte chemotactic protein-1 and its role in insulin resistance. Curr Opin Lipidol 18: 258-262, 2007.

15. Tack CJ, Stienstra R, Joosten LA and Netea MG: Inflammation links excess fat to insulin resistance: the role of the interleukin-1 family. Immunol Rev 249: 239-252, 2012.

16. Strowig T, Henao-Mejia J,Elinav E and Flavell R: Inflammasomes in health and disease. Nature 481: 278-286, 2012.

17. Benetti E, Chiazza F, Patel NS and Collino M: The NLRP3 Inflammasome as a novel player of the intercellular crosstalk in metabolic disorders. Mediators Inflamm 2013: 678627, 2013.

18. Jager J, Grémeaux T, Cormont M, Le Marchand-Brustel Y and Tanti JF: Interleukin-1beta-induced insulin resistance in adipocytes through down-regulation of insulin receptor substrate-1 expression. Endocrinology 148: 241-251, 2007.

19. Liu X, Xue R, Ji L, Zhang X, Wu J, Gu J, Zhou M and Chen S: Activation of farnesoidX receptor(FXR) protects against fructoseinduced liver steatosis via inflammatory inhibition and ADRP reduction. Biochem Biophys Res Commun 450: 117-123, 2014.

20. Dalen KT, Schoonjans K, Ulven SM, Weedon-Fekjaer MS, Bentzen TG, Koutnikova H, Auwerx J and Nebb HI: Adipose tissue expression of the lipid droplet-associating proteins S3-12 and perilipin is controlled by peroxisome proliferator-activated receptor-gamma. Diabetes 53: 1243-1252, 2004.

21. Petrighi Polidori G, Lomax MA and Docherty K: Palmitate enhances the differentiation of mouse embryonic stem cells towards white adipocyte lineages. Mol Cell Endocrinol 361: 40-50, 2012.

22. Bartels ED, Ploug T, Størling J, Mandrup-Poulsen T and Nielsen LB: Skeletal muscle apolipoprotein B expression reduces muscular triglyceride accumulation. Scand J Clin Lab Invest 74: 351-357, 2014.

23. Taniguchi CM, Emanuelli B and Kahn CR: Critical nodes in signalling pathways: Insights into insulin action. Nat Rev Mol Cell Biol 7: 85-96, 2006.

24. Yang WM, Jeong HJ, Park SY and Lee W: Induction of miR-29a by saturated fatty acids impairs insulin signaling and glucose uptake through translational repression of IRS-1 in myocytes. FEBS Lett 588: 2170-2176, 2014

25. Gao D, Madi M, Ding C, Fok M, Steele T, Ford C, Hunter L and Bing $C$ : Interleukin-1 $\beta$ mediates macrophage-induced impairment of insulin signaling in human primary adipocytes. Am J Physiol Endocrinol Metab 307: E289-E304, 2014.

26. Abdul-Ghani MA and DeFronzo RA: Pathogenesis of insulin resistance in skeletal muscle. J Biomed Biotechnol 2010: 476279, 2010.

27. Kraegen EW and Cooney GJ: Free fatty acids and skeletal muscle insulin resistance. Curr Opin Lipidol 19: 235-241, 2008.

28. Rotter V, Nagaev I and Smith U: Interleukin-6 (IL-6) induces insulin resistance in 3T3-L1 adipocytes and is, like IL-8 and tumor necrosis factor-alpha, overexpressed in human fat cells from insulin-resistant subjects. J Biol Chem 278: 45777-45784, 2003.

29. Vandanmagsar B, Youm YH, Ravussin A, Galgani JE, Stadler K, Mynatt RL, Ravussin E, Stephens JM and Dixit VD: The NLRP3 inflammasome instigates obesity-induced inflammation and insulin resistance. Nat Med 17: 179-188, 2011. 